Denisa Vitásková, Bohuslav Melichar, Marie Bartoušková, Zuzana Vlachová, David Vrána, Jana Janková, Tomáš Adam, Jarmila Juráňová, Nora Zlámalová, Lenka Kujovská Krčmová, Lenka Javorská, Dušan Klos* and Hana Študentová*

\title{
Neoadjuvant combination therapy with trastuzumab in a breast cancer patient with synchronous rectal carcinoma: a case report and biomarker study
}

https://doi.org/10.1515/pterid-2017-0017

Received August 30, 2017; accepted October 10, 2017; previously published online November 22, 2017

Abstract: We report a patient who presented with synchronous second primary human epidermal growth factor receptor (HER)-2-positive breast cancer and rectal cancer that both required simultaneous neoadjuvant therapy. A modified regimen combining anti-HER-2 monoclonal antibody trastuzumab with chemotherapy and external beam radiation was selected. An organ-preserving surgical procedure was possible both in the breast and the rectum. Citrulline decreased rapidly after the start of the treatment, and then gradually returned to pre-treatment levels after the completion of chemoradiation. Urinary neopterin concentrations exhibited a fluctuating course. Both serum neopterin and C-reactive protein concentrations were more or less stable during the initial administration of trastuzumab, paclitaxel and carboplatin and then increased steeply during chemoradiation and subsequently declined to pre-treatment levels during the weekly trastuzumab administration. Changes were observed in the serum retinol concentrations. A decline in lymphocyte counts was accompanied by marked changes in peripheral blood cell count-derived ratios. The present case report demonstrates a successful

\footnotetext{
*Corresponding authors: Dušan Klos, First Department of Surgery, Palacký University Medical School and Teaching Hospital, I.P. Pavlova 6, 779 00, Olomouc, Czech Republic, E-mail: dusan.klos@fnol.cz; and Hana Študentová, Department of Oncology, Palacký University Medical School and Teaching Hospital, I.P. Pavlova 6, 77900 Olomouc, Czech Republic, E-mail: hanastudentova@email.cz Denisa Vitásková, Marie Bartoušková and Zuzana Vlachová: Department of Oncology, Palacký University Medical School and Teaching Hospital, I.P. Pavlova 6, 77900 Olomouc, Czech Republic Bohuslav Melichar and David Vrána: Department of Oncology, Palacký University Medical School and Teaching Hospital, I.P. Pavlova 6, 77900 Olomouc, Czech Republic; and Institute of Molecular and Translational Medicine, Palacký University Medical School and Teaching Hospital, Olomouc, Czech Republic Jana Janková: Institute of Molecular and Clinical Pathology, Palacký University Medical School and Teaching Hospital, Olomouc, Czech Republic
}

combination of two neoadjuvant regimens in a patient with two synchronous different second primary tumors. Data from this case also illustrate the use of biomarkers for monitoring of intensive therapeutic regimens in medical and radiation oncology.

Keywords: breast cancer; chemoradiation; neoadjuvant therapy; neopterin; trastuzumab.

\section{Introduction}

Primary (also called neoadjuvant or induction) systemic treatment currently represents the therapy of choice in locally advanced breast carcinoma, judged inoperable based on surgical or oncological considerations. In addition, neoadjuvant therapy (mostly chemotherapy) is often administered in patients with clinically operable breast carcinoma [1, 2]. The data from randomized clinical trials as well as a meta-analysis show that the neoadjuvant or adjuvant administration of chemotherapy results in similar survival outcomes [3-6]. Neoadjuvant chemotherapy leads to a higher rate of breast saving surgery and, at the same time, allows for the evaluation of sensitivity of the tumor in an individual patient to a defined chemotherapy regimen [6].

Tomáš Adam: Institute of Molecular and Translational Medicine, Palacký University Medical School and Teaching Hospital, Olomouc, Czech Republic; and Department of Clinical Biochemistry, Palacký University Medical School and Teaching Hospital, Olomouc, Czech Republic

Jarmila Juráňová: Department of Hemato-Oncology, Palacký University Medical School and Teaching Hospital, Olomouc, Czech Republic

Nora Zlámalová: First Department of Surgery, Palacký University Medical School and Teaching Hospital, I.P. Pavlova 6, 77900 Olomouc, Czech Republic

Lenka Kujovská Krčmová and Lenka Javorská: Third Department of Medicine (Gerontology and Metabolic Care), Charles University Teaching Hospital, Hradec Králové, Czech Republic; and Department of Analytical Chemistry, Charles University School of Pharmacy, Hradec Králové, Czech Republic 
In the past decade, there has been a resurge of interest in cancer immunology [7]. The idea to harness the immune system for cancer cure is not new. The recent progress in the immunotherapy of tumors has coincided with the advent of targeted therapy [7]. One of the two fundamental approaches to design new drugs with targeted mechanism of action is the creation of monoclonal antibodies against tumor cell targets. While monoclonal antibodies were originally used in this setting solely to achieve selective inhibition of a defined molecular target, it later became evident that some monoclonal antibodies envisaged to block molecules associated with tumor growth and progression also exert antitumor activity through the activation of the immune system by antibody-dependent cell-mediated cytotoxicity $[8,9]$.

Tumors in about $15 \%$ of patients with breast cancer are characterized by an over-expression of human epidermal growth factor receptor (HER)-2. The over-expression of HER-2 is associated with aggressive biological behavior, and, before the era of targeted therapy, the outcome of patients with HER-2-positive breast carcinoma was poor. The introduction of trastuzumab, a humanized monoclonal antibody against HER-2, has transformed the natural history of HER-2-positive breast carcinoma, and anti-HER-2 therapy is currently the standard component of treatment in the adjuvant, neoadjuvant or metastatic settings in these patients [10].

Improved prognosis of cancer patients is accompanied by an increased incidence of second primary tumors. A synchronous presentation of two different primary tumors may present a challenge with regard to diverse systemic therapy regimens that may need to be administered simultaneously or sequentially. We report here a patient who presented with synchronous second primary HER-2-positive breast cancer and rectal cancer that both required neoadjuvant therapy.

\section{Case description}

A 44-year-old patient presented in January 2014 with a lump in the left breast. The patient had a history of rightsided breast cancer that was treated in December 2004 by lumpectomy and adjuvant chemotherapy (a total of six cycles of the combination of doxorubicin, cyclophosphamide and 5-fluorouracil), adjuvant radiation and hormonal therapy in 2005. A histological examination of the tumor in the left breast revealed hormone receptor-negative, HER-2 positive carcinoma. The family history was negative, and a molecular genetic analysis did not reveal mutations in the $B R C A 1$ or $B R C A 2$ genes. Laboratory examinations at the time of the presentation in January 2014 revealed anemia and an increased serum concentration of carcinoembryonic antigen (CEA) of $92.7 \mu \mathrm{g} / \mathrm{L}$ (reference range 0-4.7 $\mu \mathrm{g} / \mathrm{L}$ ). Because of the high prior cumulative dose of doxorubicin $\left(300 \mathrm{mg} / \mathrm{m}^{2}\right)$ the treatment with the combination of trastuzumab, paclitaxel and carboplatin administered in a weekly regimen was planned. Before initiation of treatment, the clarification of the cause of anemia was warranted. A stenosing rectal tumor $8 \mathrm{~cm}$ from the anal verge was diagnosed in February 2014. The diagnosis of adenocarcinoma of the rectum was confirmed by a histological examination. Positron emission tomography/computed tomography (PET/CT) did not reveal any distant metastases, but because of the phenotype and clinical stage of the tumor in the left breast (T2NOM0) and the clinical stage of the rectal carcinoma (T3NOM0) neoadjuvant strategy was selected for both tumors, neoadjuvant systemic chemotherapy for the breast cancer and neoadjuvant chemoradiation for the rectal carcinoma.

However, in the present case the neoadjuvant strategy required important modification of the therapeutic regimen. Because of prior exposure to anthracyclines and left-sided location, anthracyclines could not be considered for the treatment of breast cancer. Most importantly, it was necessary to find a regimen that would have activity for both tumors. In March 2014 (starting on March 3) while the preparations and planning were done for radiotherapy the patient was treated with four weekly doses of trastuzumab ( $4 \mathrm{mg} / \mathrm{kg}$ loading dose, then $2 \mathrm{mg} / \mathrm{kg}$ ), paclitaxel $\left(90 \mathrm{mg} / \mathrm{m}^{2}\right)$ and carboplatin (area under the curve 2). With the start of external beam radiation ( $45 \mathrm{~Gy}$ in 25 fractions to the pelvis, boost to the tumor to the total dose of $50.4 \mathrm{~Gy}$ ) from April 1, 2014, trastuzumab (2 mg/kg) was combined with weekly cisplatin $\left(20 \mathrm{mg} / \mathrm{m}^{2}\right)$ and continuous infusion of 5 -fluorouracil $\left(200 \mathrm{mg} / \mathrm{m}^{2} /\right.$ day $)$. After 1 week of the combined regimen, the therapy was complicated by grade 2 diarrhea. Continuous 5 -fluorouracil was interrupted, and trastuzumab was solely administered on April 8 and April 16. After diarrhea was controlled by supportive measures including oral loperamide, the administration of the combined regimen of trastuzumab, cisplatin and 5-fluorouracil was continued until May 21 when the chemotherapy was terminated (1 week after the completion of radiotherapy). Weekly trastuzumab was continued subsequently until surgery. On July 29, 2014 the patient underwent quadrantectomy with sentinel node biopsy. A pathological examination of the surgical specimen revealed pathological complete response. No tumor was detected in the sentinel lymph node. Subsequently, on August 6, 2014 the patient underwent low resection of the 
rectum. The resection specimen exhibited marked regressive changes in the tumor with healing ulceration and small extent of the focal residual tumor nests. Ten lymph nodes were examined, all without evidence of tumor. The postoperative course was uneventful and the patient quickly returned to daily activities.

Postoperatively, adjuvant radiotherapy to the breast (50 Gy in 25 fractions) was administered together with brachytherapy boost to the tumor bed (12 Gy in a single fraction), and trastuzumab was continued for a total duration of 1 year as subcutaneous injection of $600 \mathrm{mg}$ every 3 weeks. At the last control on September 27, 2017, 3.5 years after the initial diagnosis the patient was well and without evidence of disease. Anemia gradually improved, and hemoglobin concentration at the last control was $135 \mathrm{~g} / \mathrm{L}$.

The treatment course was reflected in the concentration of biomarkers that were determined as described earlier [11-15]. These biomarkers were determined in this patient as part of a research project approved by the Institutional Ethical Committee, and the patient signed informed consent. Citrulline decreased rapidly from a pre-treatment concentration of 38-15 $\mu \mathrm{mol} / \mathrm{L}$ on March 31 and after temporary stabilization to a nadir of $12 \mu \mathrm{mol} / \mathrm{L}$ (Figure 1A). Citrulline concentrations gradually returned to pre-treatment levels after the completion of chemoradiation during the administration of weekly trastuzumab. Urinary neopterin concentrations exhibited a fluctuating course (Figure 1B). Both serum neopterin (Figure 1C) and C-reactive protein (CRP; Figure 1D) concentrations were more or less stable during the initial administration of trastuzumab, paclitaxel and carboplatin and then increased steeply during chemoradiation to a marked peak in May 2014 that coincided with the last fractions of chemoradiation with a subsequent decline to pretreatment levels during the weekly trastuzumab administration. Serum kynurenine (Figure 2A) increased, serum tryptophan decreased (Figure 2B) and the kynurenine/ tryptophan ratio (Figure $2 \mathrm{C}$ ) increased during the course of therapy. At the time of peak neopterin or CRP levels and kynurenine/tryptophan ratio and nadir citrulline and tryptophan concentrations the patient complained about abdominal cramps, but had no other symptoms.

Serum retinol concentrations exhibited a fluctuating course with a decline during chemoradiation and subsequent recovery (Figure 2D). $\alpha$-Tocopherol concentrations exhibited only mild fluctuations (Figure 3A), while serum 25-hydroxy vitamin D3 concentrations declined during chemoradiation with subsequent recovery (Figure 3B).

\section{A}

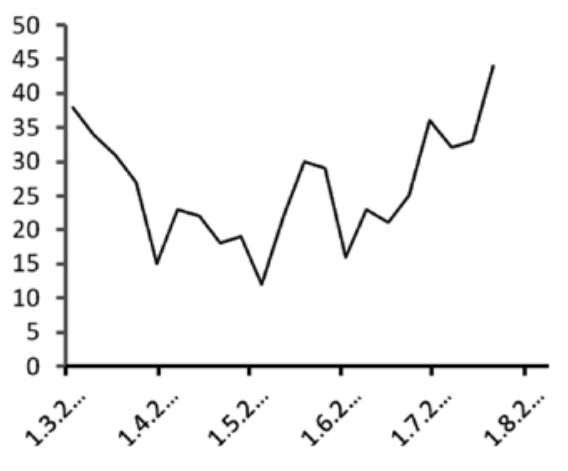

C

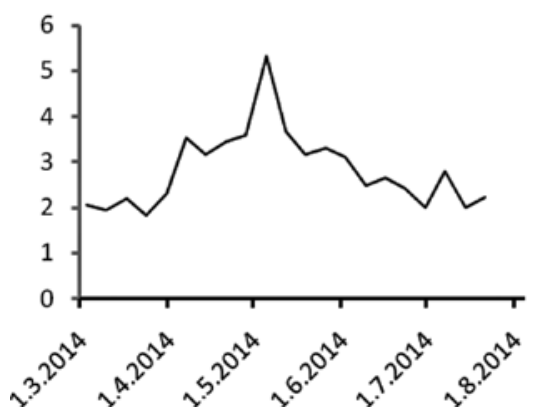

B

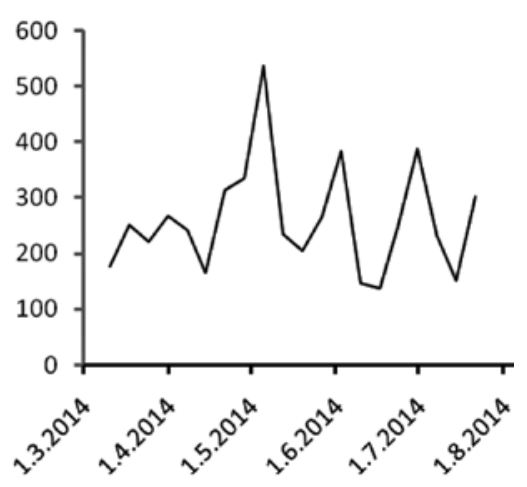

D

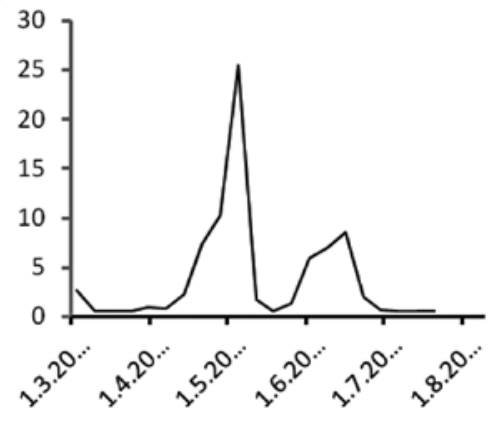

Figure 1: Citrulline, neopterin and C-reactive protein during the course of treatment. (A) Plasma citrulline (in $\mu \mathrm{mol} / \mathrm{L}$ ), (B) urinary neopterin (in $\mu \mathrm{mol} / \mathrm{mol}$ creatinine), (C) serum neopterin (in $\mu \mathrm{g} / \mathrm{L}$ ) and (D) C-reactive protein (in $\mathrm{mg} / \mathrm{L}$ ) concentrations measured in weekly intervals during the course of treatment. 
A

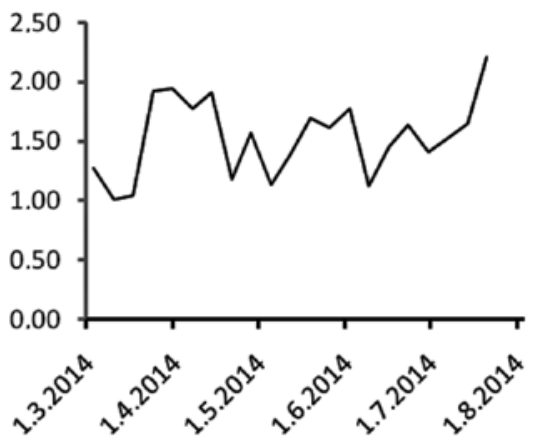

C

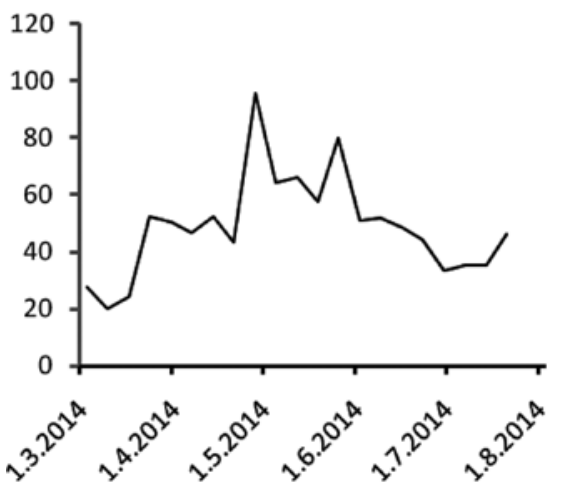

B

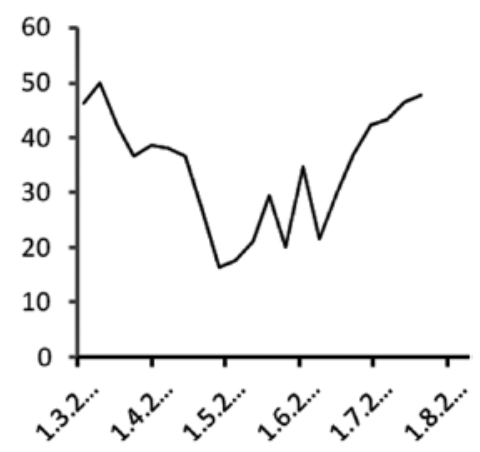

D

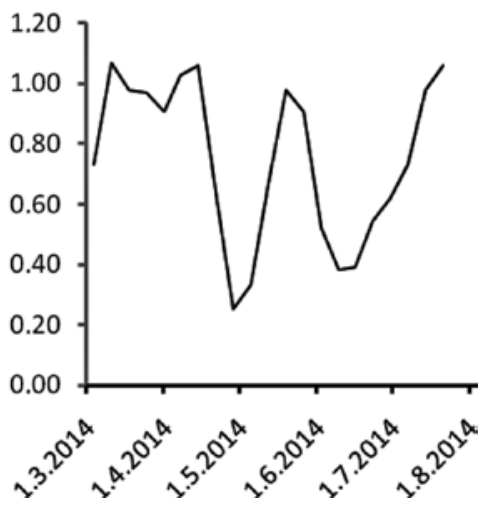

Figure 2: Kynurenine, tryptophan and retinol during the course of treatment.

(A) Serum kynurenine concentrations (in $\mu \mathrm{mol} / \mathrm{L}$ ), (B) serum tryptophan concentrations (in $\mu \mathrm{mol} / \mathrm{L}$ ), (C) serum kynurenine/tryptophan ratios (in $\mathrm{mmol} / \mathrm{mol}$ ) and (D) serum retinol concentrations (in $\mu \mathrm{mol} / \mathrm{L}$ ) measured in weekly intervals during the course of treatment.

The hemoglobin concentration was low throughout the course of treatment (Figure 3C) and a transfusion was necessary in July 2014 before the surgery. Ferritin and iron were also low throughout the course of treatment (data not shown), consistently with the diagnosis of iron deficiency anemia resulting from chronic blood loss. Concentrations of transferrin were within the normal range and soluble transferrin receptor levels were mildly elevated throughout the course of therapy (data not shown). The CEA concentration declined to normal levels $(<4.7 \mu \mathrm{g} / \mathrm{L})$ after the treatment.

The peripheral blood lymphocyte counts declined steeply during the initial weeks of the treatment and remained at low levels below or around 400 cells per $\mu \mathrm{L}$ subsequently (Figure 3D). The decrease of absolute lymphocyte counts was reflected in increasing neutrophil-to-lymphocyte ratio (Figure $4 \mathrm{~A}$ ) and decreasing lymphocyte-to-monocyte ratio (Figure 4B). The plateletto-lymphocyte ratio was stable during the administration of paclitaxel/carboplatin combination and subsequently increased steeply (Figure 4C). The systemic inflammatory index (systemic immune-inflammation index) [14] exhibited a similar course as the platelet-to-lymphocyte ratio (Figure 4D).

\section{Discussion}

The present case report demonstrates a successful use of a neoadjuvant regimen targeting two synchronous second primary tumors, HER-2-positive breast carcinoma and rectal carcinoma. Although second primary malignancies are common, synchronous presentation of two different primary tumors is a relatively rare event even for the most frequent malignancies like breast cancer or colorectal carcinoma. Therefore, case reports like the present one remain the only source of information on the management of patients with this rare presentation as it would not be possible to organize prospective trials.

Different biology of rectal carcinoma and HER-2-positive breast carcinoma is also reflected in different regimens used in the neoadjuvant setting [16, 17]. In fact, the standard neoadjuvant regimens for these two tumors have no common features. Furthermore, history of exposure to anthracycline excluded the possibility of using anthracyclines because of the risk of cardiac toxicity that would be further increased by the need for radiotherapy [18]. The present case report demonstrates how two seemingly disparate regimens of neoadjuvant therapy can be 
A

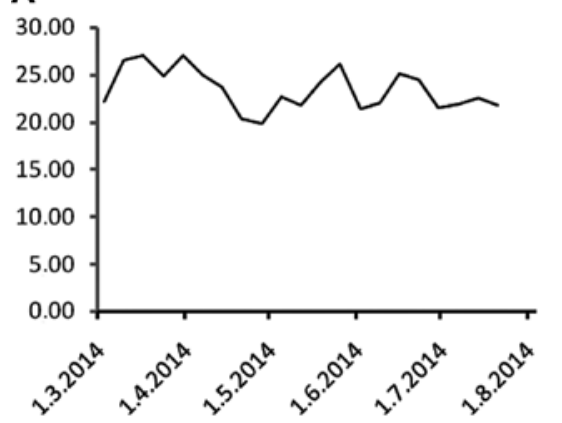

C

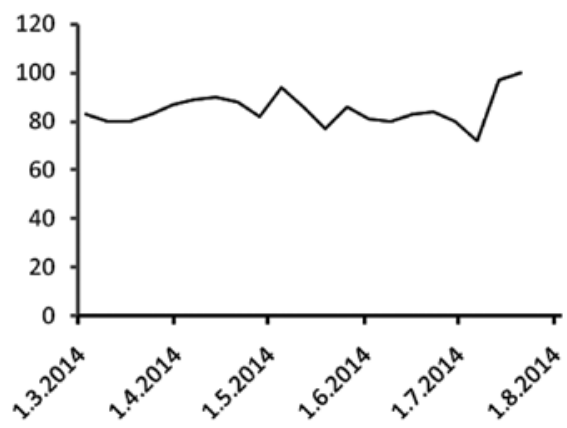

B

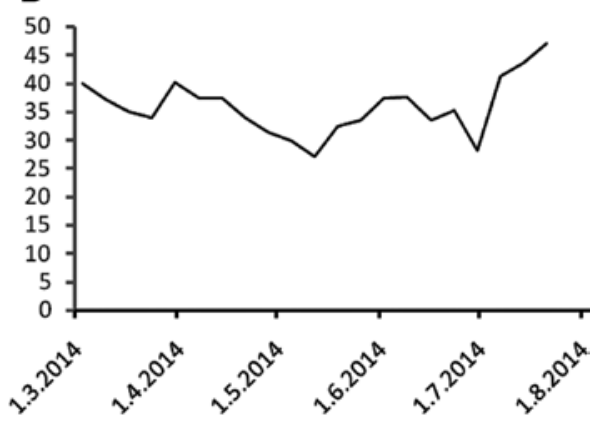

D

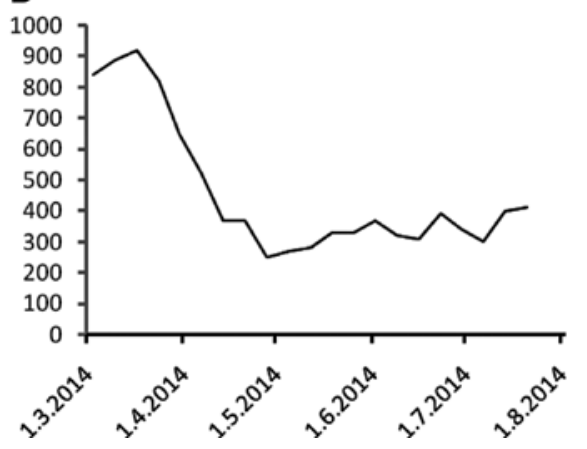

Figure 3: $\alpha$-Tocopherol, 25-hydroxy vitamin D3, hemoglobin and lymphocyte counts during the course of treatment.

(A) Serum $\alpha$-tocopherol concentrations (in $\mu \mathrm{mol} / \mathrm{L}$ ), (B) serum 25-hydroxy vitamin D3 concentrations (in nmol/L), (C) hemoglobin concentrations (in $\mathrm{g} / \mathrm{L}$ ) and $(\mathrm{D})$ peripheral blood lymphocyte counts (as cells/ $\mu \mathrm{L}$ ) measured in weekly intervals during the course of treatment.

A

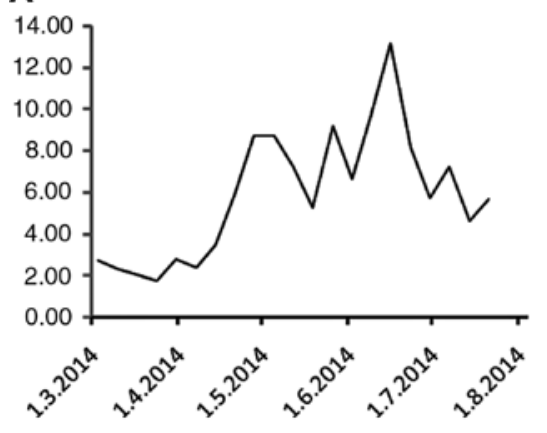

C

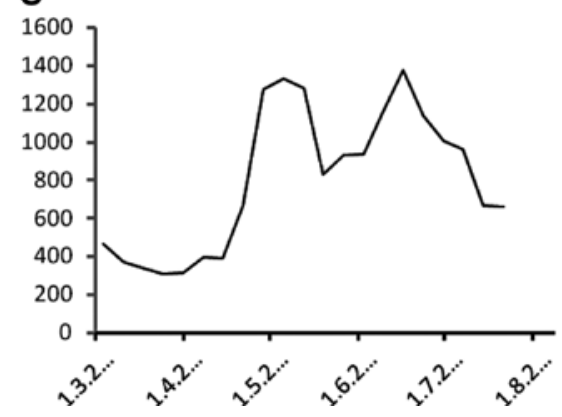

B

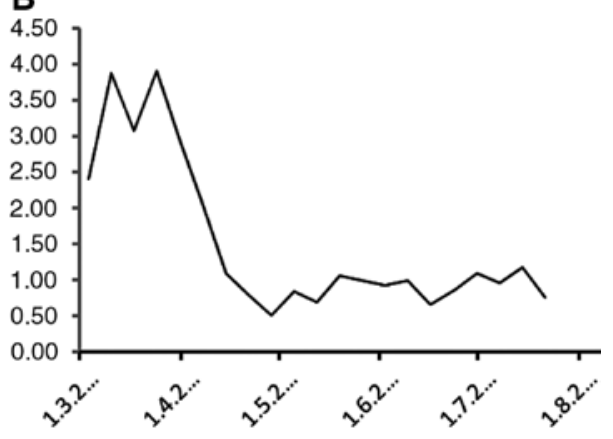

D

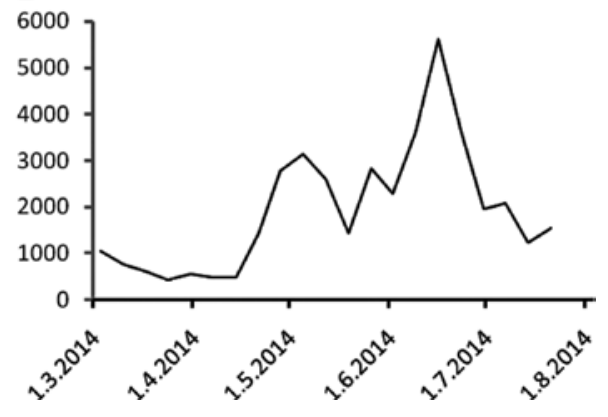

Figure 4: Peripheral blood cell count-derived ratios during the course of treatment.

(A) The neutrophil-to-lymphocyte ratio, (B) the lymphocyte-to-monocyte ratio, (C) the platelet-to-lymphocyte ratio and (D) the systemic inflammatory index determined in weekly intervals during the course of treatment. 
successfully combined. After a short introductory course of the combination of trastuzumab with paclitaxel and carboplatin, the chemotherapy backbone was switched to 5-fluouracil and cisplatin while continuing weekly trastuzumab administration. Paclitaxel and carboplatin are regarded as inactive agents in rectal carcinoma while both 5 -fluorouracil and cisplatin have high activity in breast cancer $[19,20]$. The standard regimen of chemoradiation consists of continuous 5-fluorouracil administration. Cisplatin was added here mainly to increase activity of the 5 -fluorouracil plus trastuzumab combination against breast cancer.

A pathological complete response was obtained in the breast. The pathological complete response rate in HER2-positive breast cancer is close to 50\% [21]. Importantly, a pathological complete response is associated with an improved prognosis.

Laboratory medicine is currently playing an increasingly important role in the management of cancer patients [22]. The present report illustrates the utilization of biomarkers in monitoring the activation of the immune system and toxicity of the therapy. The systemic administration of anticancer therapy is almost always accompanied by side-effects because cytotoxic drugs damage not only tumor cells, but virtually any tissue with high mitotic activity. Effects on tissues with highest mitotic activity, i.e. bone marrow and gastrointestinal mucosa, are associated with the two most common adverse events accompanying the administration of cytotoxic chemotherapy [23]. While laboratory methods can determine peripheral blood cell count as continuous variables allowing for a graded assessment and differential response to the changes induced by the treatment, the assessment of gastrointestinal toxicity still depends almost exclusively on the data reported by the patients that may be affected by subjective factors and are often impossible to verify. Different laboratory biomarkers have been introduced in the assessment of gastrointestinal toxicity of systemic anticancer agents, including the measurement of intestinal permeability or monitoring of citrulline concentrations, as in the present report [11, 2426]. Despite promising results of intestinal permeability, this method is not well suited for routine clinical practice. Citrulline has been proposed as another biomarker of gastrointestinal toxicity of chemotherapy and/or radiation that is much more practical in clinical routine [11, $27,28]$.

In the present case, the administration of the combined regimen was accompanied by a gradual decrease of plasma citrulline that was accompanied by a rise of serum and urinary neopterin concentrations as well as serum CRP. The highest concentrations of inflammatory biomarkers were observed at the end of chemoradiation.

It is well known that chemotherapy induces activation of systemic inflammatory and immune responses [29-31]. Systemic inflammatory or immune response can be assessed by determining circulating cytokine concentrations, but sometimes marked fluctuations of cytokine concentrations may occur even within a short time frame. Moreover, the investigation of dynamics of immune or inflammatory response is compromised by the need for repeated venepuncture and associated office visits. Neopterin may be determined not only in serum, but also in other body fluids, including urine. The measurement of urinary neopterin for the assessment of systemic immune and inflammatory response presents an advantage as repeated measurements over a prolonged period of time are easily possible.

Neopterin is produced by macrophages from guanosine triphosphate (GTP). Interferon-gamma (IFN- $\gamma$ ) induces GTP cyclohydrolase I that catalyzes the production of neopterin. The concentrations of IFN- $\gamma$ reflect the systemic immune response, but the production of IFN- $\gamma$ is enhanced by pro-inflammatory cytokines, e.g. interleukin-1 or interleukin-6 that, consequently, also enhance neopterin production. Neopterin concentrations are therefore thought to reflect both systemic immune and inflammatory responses [32]. Serum or urinary neopterin can be used as a biomarker of systemic immune and inflammatory responses in a wide range of disorders including malignancies, viral infections, transplant rejection, advanced age or atherosclerosis and its complications [32-40]. Neopterin is stable in refrigerated samples for up to 2 weeks, and samples for repeat assessment may be collected and stored by the patient between regular office visits with the need for repeated puncture circumvented. It has been demonstrated that urinary neopterin concentrations in cancer patients are stable in the absence of complications [41]. A rise of neopterin concentrations when followed daily in patients after organ transplantation represents an early biomarker of acute complications [32]. Similarly, it has been reported in cancer patients undergoing daily monitoring of urinary neopterin concentrations that increased concentrations preceded complications while a decrease indicated tumor control [41]. In patients with advanced cancer increased neopterin concentrations have been associated with immune dysfunction [42-45].

Surprisingly, the literature on neopterin as a biomarker of systemic immune and inflammatory responses in patients with breast cancer is relatively limited. Increased urinary neopterin concentrations were reported in $<20 \%$ of breast cancer patients $[40,46]$. Neopterin 
concentrations correlated with tumor grade and the presence of advanced disease [40, 46], and increased neopterin was indicative of poor prognosis in both univariate and multivariate analyses [46]. In another report, slightly elevated serum neopterin concentrations were observed in breast cancer survivors with chronic fatigue [47]. A rise of neopterin concentrations has been documented in breast cancer patients treated with doxorubicin/paclitaxel combination chemotherapy [48].

Another biomarker of the activation of the immune system is kynurenine and kynurenine/tryptophan ratio. The increase in the kynurenine/tryptophan ratio in the present case accompanied the rise in neopterin concentrations. Although tryptophan depletion inhibits tumor growth and kynurenine [49], at high concentrations, and has some direct antitumor activity [50], it is now generally accepted that the increased kynurenine concentration and tryptophan depletion are immunosuppressive in cancer patients [51].

Cancer pathogenesis and the administration of anticancer treatment are associated with oxidative stress [52-54]. Moreover, antioxidant balance is thought to be disturbed in association with the toxicity of anticancer therapy. $\alpha$-Tocopherol is the major antioxidant in the serum [55]. Perturbations of the oxidant-antioxidant equilibrium including $\alpha$-tocopherol were shown to be associated with the toxicity of radiotherapy $[56,57]$ or chemotherapy [58]. In prior reports, a decrease of serum $\alpha$-tocopherol concentrations has been noted during the administration of systemic chemotherapy $[52,53,59,60]$. A decrease of serum retinol concentrations has also been reported in patients with advanced tumors, and the decline of $\alpha$-tocopherol and retinol concentrations in cancer patients correlated with biomarkers of systemic inflammatory response like CRP $[61,62]$. In the present case, serial serum retinol concentrations were inversely associated with changes in serum or urinary neopterin or CRP concentrations, similar to an earlier report [63] while no marked trend was observed for $\alpha$-tocopherol and 25-hydroxy vitamin D3.

The increase in the biomarkers of systemic immune and inflammatory response was accompanied by lymphopenia that was reflected in the high neutrophil-tolymphocyte ratio, platelet-to-lymphocyte ratio or systemic inflammatory index and low lymphocyte-to-monocyte ratio. The rise of platelet-to-lymphocyte ratio was delayed because of the toxicity of carboplatin administered during the initial phase of the treatment.

In conclusion, the present case report demonstrates a successful combination of two neoadjuvant regimens in a patient with two synchronous different second primary tumors. Data from this case also illustrate the use of biomarkers for monitoring intensive therapeutic regimens in medical and radiation oncology.

Acknowledgment: This study was supported by the grant of the Czech Health Research Council 16-32030A.

Conflict of interest statement: Bohuslav Melichar, David Vrána, Denisa Vitásková and Hana Študentová received honoraria for lectures from Roche; Bohuslav Melichar received honoraria from advisory boards.

\section{References}

1. Estevez LG, Gradishar WJ. Evidence-based use of neoadjuvant taxane in operable and inoperable breast cancer. Clin Cancer Res 2004;10:3249-61.

2. Kaufmann M, Hortobagyi GN, Goldhirsch A, Scholl S, Makris A, Valagussa $P$, et al. Recommendations from an international expert panel on the use of neoadjuvant (primary) systemic treatment of operable breast cancer: an update. J Clin Oncol 2006;24:1940-9.

3. Fisher B, Brown A, Mamounas E, Wieand S, Robidoux A, Margolese RG, et al. Effect of preoperative chemotherapy on localregional disease in women with operable breast cancer: findings from National Surgical Adjuvant Breast and Bowel Project B-18. J Clin Oncol 1997;15:2483-93.

4. Fisher B, Bryant J, Wolmark N, Mamounas E, Brown A, Fisher ER, et al. Effect of preoperative chemotherapy on the outcome of women with operable breast cancer. J Clin Oncol 1998;16:2672-85.

5. van der Hage JA, van de Velde CJ, Julien JP, Tubiana-Hulin M, Vandervelden C, Duchateau L. Preoperative chemotherapy in primary operable breast cancer: results from the European Organization for Research and Treatment of Cancer trial 10902. J Clin Oncol 2001;19:4224-37.

6. Mauri D, Pavlidis N, loannidis JP. Neoadjuvant versus adjuvant systemic treatment in breast cancer: a meta-analysis. J Natl Cancer Inst 2005;97:188-94.

7. Kirkwood JM, Butterfield LH, Tarhnini AA, Zarour H, Kalinski P, Ferrone S. Immunotherapy of cancer in 2012. CA Cancer J Clin 2012;62:309-35.

8. Musolino A, Naldi N, Bortesi B, Pezzuolo D, Capelletti M, Missale $\mathrm{G}$, et al. Immunoglobulin $\mathrm{G}$ fragment $C$ receptor polymorphisms and clinical efficacy of trastuzumab-based therapy in patients with HER-2/neu-positive metastatic breast cancer. J Clin Oncol 2008;26:1789-96.

9. Zhang W, Gordon M, Schultheis AM, Yang DY, Nagashima F, Azuma M, et al. ECGR2A and FCGR3A polymorphisms associated with clinical outcome of epidermal growth factor receptorexpressing metastatic colorectal cancer patients treated with single-agent cetuximab. J Clin Oncol 2007;25:3712-8.

10. Boekhout AH, Beijnen JH, Schellens JH. Trastuzumab. Oncologist 2011;16:800-10.

11. Zezulova M, Bartouskova M, Hlidkova E, Adam T, Kujovska Krcmova L, Cervinkova B, et al. Citrulline as a biomarker of gastrointestinal toxicity in patients with rectal carcinoma treated with chemoradiation. Clin Chem Lab Med 2016;54:305-14. 
12. Pilka R, Marek R, Adam T, Kudela M, Ondrova D, Neubert D, et al. Systemic inflammatory response after open, laparoscopic and robotic surgery in endometrial cancer patients. Anticancer Res 2016;36:2909-22.

13. Zezulova M, Bartouskova M, Hlidkova E, Juranova J, Cervinkova $B$, Kasalova E, et al. Prognostic significance of serum and urinary neopterin concentrations in patients with rectal carcinoma treated with chemoradiation. Anticancer Res 2016;36:287-92.

14. Hu B, Yang XR, Xu Y, Sun YF, Sun C, Guo W, et al. Systemic immune-inflammation index predicts prognosis of patients after curative resection for hepatocellular carcinoma. Clin Cancer Res 2014;20:6212-22.

15. Plíšek J, Kujovská Krčmová L, Aufartová J, Morales TV, Esponda $\mathrm{SM}$, Oros R, et al. New approach for the clinical monitoring of 25-hydroxyvitamin D3 and 25-hydroxyvitamin D2 by ultra high performance liquid chromatography with MS/MS based on the standard reference material 972. J Sep Sci 2013;38:3702-8.

16. Buzdar AU, Ibrahim NK, Francis D, Booser DJ, Thomas ES, Theriault RL, et al. Significantly higher pathologic complete remission rate after neoadjuvant therapy with trastuzumab, paclitaxel, and epirubicin chemotherapy: results of a randomized trial in human epidermal growth factor receptor 2-positive operable breast cancer. J Clin Oncol 2005;23:3676-85.

17. Sauer R, Becker H, Hohenberger W, Rodel C, Wittekind C, Fietkau $\mathrm{R}$, et al. Preoperative versus postoperative chemoradiotherapy for rectal cancer. N Engl J Med 2004;351:1731-40.

18. $\mathrm{Ng} \mathrm{R,} \mathrm{Bettrer} \mathrm{N,} \mathrm{Green} \mathrm{MD.} \mathrm{Anticancer} \mathrm{agents} \mathrm{and} \mathrm{cardiotoxicity.}$ Semin Oncol 2006;14:2-14.

19. Vogel A, Hofheinz RD, Kubicka S, Arnold D. Treatment decisions in metastatic colorectal cancer - Beyond first and second line combination therapies. Cancer Treat Rev 2017;59:54-60.

20. Al-Tweigeri T, AlSayed A, Alawadi S, Ibrahim M, Ashour W, Jaafar $\mathrm{H}$, et al. A multicenter prospective phase II trial of neoadjuvant epirubicin, cyclophosphamide, and 5-fluorouracil (FEC100) followed by cisplatin-docetaxel with or without trastuzumab in locally advanced breast cancer. Cancer Chemother Pharmacol 2016;77:147-53.

21. Ismael G, Hegg R, Muehlbauer S, Heinzmann D, Lum B, Kim $\mathrm{SB}$, et al. Subcutaneous versus intravenous administration of (neo)adjuvant trastuzumab in patients with HER2-positive, clinical stage I-III breast cancer (HannaH study): a phase 3, open-label, multicentre, randomised trial. Lancet Oncol 2012;13:869-78.

22. Melichar B. Laboratory medicine and medical oncology: the tale of two Cinderellas. Clin Chem Lab Med 2013;51:99-112.

23. Melichar B, Dvorak J, Hyspler R, Zadak Z. Intestinal permeability in the assessment of intestinal toxicity of cytotoxic agents. Chemotherapy 2005;51:336-8.

24. Melichar B, Hyspler R, Ticha A, Kalabova H, Vitaskova D, Zezulova $M$, et al. Intestinal permeability in patients with metastatic colon cancer treated with patupilone. Clin Chem Lab Med 2014;52:1649-55.

25. Dvorak J, Melichar B, Hyspler R, Krcmova L, Urbanek L, Kalabova $\mathrm{H}$, et al. Intestinal permeability, vitamin A absorption, alpha-tocopherol, and neopterin in patients with rectal carcinoma treated with chemoradiation. Med Oncol 2010;27:690-6.

26. Melichar B, Kohout P, Bratova M, Solichova D, Kralickova P, Zadak Z. Intestinal permeability in patients with chemotherapyinduced stomatitis. J Cancer Res Clin Oncol 2001;127:314-8.
27. van Vliet MJ, Tissing WJ, Rings EH, Koetse HA, Stellaard F, Kamps WA, et al. Citrulline as a marker for chemotherapy induced mucosal barrier injury in pediatric patients. Pediatr Blood Cancer 2009;53:1188-94.

28. Lutgens LC, Blijlevens NM, Deutz NE, Donnelly JP, Lambin P, de Pauw BE. Monitoring myeloablative therapy-induced small bowel toxicity by serum citrulline concentration. A comparison with sugar permeability tests. Cancer 2005;103:191-9.

29. Pusztai L, Mendoza TR, Reuben JM, Martinez MM, Willey JS, Lara J, et al. Changes in plasma levels of inflammatory cytokines in response to paclitaxel chemotherapy. Cytokine 2004;25: 94-102.

30. Tsavaris N, Kosmas C, Vadiaka M, Kanelopoulos P, Boulamatsis $D$. Immune changes in patients with advanced breast cancer undergoing chemotherapy with taxanes. Br J Cancer 2002;87:21-7.

31. Melichar B, Urbanek L, Krcmova L, Kalabova H, Melicharova K, Malirova E, et al. Urinary neopterin, hemoglobin and peripheral blood cell counts in breast carcinoma patients treated with dose-dense chemotherapy. Anticancer Res 2008;28:2389-96.

32. Wachter H, Fuchs D, Hausen A, Reibnegger G, Werner ER. Neopterin as marker for activation of cellular immunity: immunologic basis and clinical application. Adv Clin Chem 1989;27:81-141.

33. Melichar B, Gregor J, Solichova D, Lukes J, Tichy M, Pidrman V. Increased urinary neopterin in acute myocardial infarction. Clin Chem 1994;40:338-9.

34. Solichova D, Melichar B, Blaha V, Klejna M, Vavrova J, Palicka $\mathrm{V}$, et al. Biochemical profile and survival in nonagenarians. Clin Biochem 2001;34:563-9.

35. Melichar B, Solichova D, Melicharova K, Malirova E, Cermanova $M$, Zadak Z. Urinary neopterin in patients with advanced colorectal carcinoma. Int J Biol Markers 2006;21:190-8.

36. Reibnegger G, Aichberger C, Fuchs D, Hausen A, Spielberger $M$, Werner ER, et al. Posttransplant neopterin excretion in renal allograft recipients - a reliable diagnostic aid for acute rejection and a predictive marker of long-term graft survival. Transplantation 1991;52:58-63.

37. Fuchs D, Hausen A, Reibnegger G, Werner ER, Dierich MP, Wachter H. Neopterin as a marker for activated cell mediated immunity: application in HIV infection. Immunol Today 1988;9:150-5.

38. Weiss G, Willeit J, Kiechl S, Fuchs D, Jarosch E, Oberhollenzer F, et al. Increased concentrations of neopterin in carotid atherosclerosis. Atherosclerosis 1994;106:263-71.

39. Grammer TB, Fuchs D, Boehm BO, Winkelmann BR, Maerz W. Neopterin as a predictor of total and cardiovascular mortality in individuals undergoing angiography in the Ludwigshafen Risk and Cardiovascular Health Study. Clin Chem 2009;55:1135-46.

40. Melichar B, Solichová D, Freedman RS. Neopterin as an indicator of immune activation and prognosis in patients with gynecological malignancies. Int J Gynecol Cancer 2006;16:240-52.

41. Melichar B, Kalabova H, Urbanek L, Malirova E, Solichova D. Serial urinary neopterin measurements reflect the disease course in patients with epithelial ovarian carcinoma treated with paclitaxel/platinum. Pteridines 2007;18:1-7.

42. Melichar B, Jandik P, Krejsek J, Solichova D, Drahosova M, Skopec F, et al. Mitogen-induced lymphocyte proliferation and systemic immune activation in cancer patients. Tumori 1996;82:218-20. 
43. Melichar B, Touskova M, Solichova D, Kralickova P, Kopecky O. CD4+ T-lymphocytopenia and systemic immune activation in patients with primary and secondary liver tumours. Scand J Clin Lab Invest 2001;61:363-70.

44. Melichar B, Nash MA, Lenzi R, Platsoucas CD, Freedman RS. Expression of costimulatory molecules CD80 and CD86 and their receptors CD28, CTLA-4 on malignant ascites CD3+ tumor infiltrating lymphocytes (TIL) from patients with ovarian and other types of peritoneal carcinomatosis. Clin Exp Immunol 2000;119:19-27.

45. Melichar B, Savary C, Kudelka AP, Verschraegen C, Kavanagh JJ, Edwards $C L$, et al. Lineage-negative human leukocyte antigen$\mathrm{DR}+$ cells with the phenotype of undifferentiated dendritic cells in patients with carcinoma of the abdomen and pelvis. Clin Cancer Res 1998;4:799-809.

46. Murr C, Bergant A, Widschwendter M, Heim K, Schrocksnadel $\mathrm{H}$, Fuchs $\mathrm{D}$. Neopterin is an independent prognostic variable in females with breast cancer. Clin Chem 1999;45:1998-2004.

47. Bower JE, Ganz PA, Aziz N, Fahey JL, Cole SW. T-cell homeostasis in breast cancer survivors with persistent fatigue. J Natl Cancer Inst 2003;95:1165-8.

48. Melichar B, Solichova D, Melicharova K, Cermanova M, Urminska H, Ryska A. Systemic immune activation, anemia and thrombocytosis in breast cancer patients treated by doxorubicin and paclitaxel. Pteridines 2006;17:107-14.

49. Burke F, Knowles RG, East N, Balkwill FR. The role of indoleamine 2,3-dioxygenase in the anti-tumour activity of human interferongama in vivo. Int J Cancer 1995;60:115-22.

50. Melichar B, Ferrandina G, Verschraegen CF, Loercher A, Abbruzzese JL, Freedman RS. Growth inhibitory effects of aromatic fatty acids on ovarian tumor cell lines. Clin Cancer Res 1998;4:3069-76.

51. Friberg M, Jennings R, Alsarraj M, Dessureault S, Cantor A, Extermann M, et al. Indoleamin 2,3-dioxygenase contributes to tumor cell evasion of T cell-mediated rejection. Int J Cancer 2002;101:151-5.

52. Faber M, Coudray C, Hida H, Mousseau M, Favier A. Lipid peroxidation products, and vitamin and trace element status in patients with cancer before and after chemotherapy, including adriamycin. A preliminary study. Biol Trace Elem Res 1995;47:117-23.
53. Faure H, Coudray C, Mousseau M, Ducros V, Douki T, Bianchini F, et al. 5-Hydroxymethyluracil excretion, plasma TBARS and plasma antioxidant vitamins in adriamycin-treated patients. Free Radic Biol Med 1996;20:979-83.

54. Weijl NI, Hopman GD, Wipkink-Bakker A, Lentjes EG, Berger HM, Cleton FJ, et al. Cisplatin combination chemotherapy induces a fall in plasma antioxidants of cancer patients. Ann Oncol 1998;9:1331-7.

55. Debier C, Larondelle Y. Vitamins A and E: metabolism, roles and transfer to offspring. Br J Nutr 2005;93:153-74.

56. Delanian S, Porcher R, Balla-Mekias S, Lefaix JL. Randomized, placebo-controlled trial of combined pentoxifylline and tocopherol for regression of superficial radiation-induced fibrosis. J Clin Oncol 2003;21:2545-50.

57. Erhola M, Nieminen MM, Ojala A. Human plasma antioxidant capacity during radiotherapy for lung cancer: a clinical study. J Exp Clin Cancer Res 1998;17:325-30.

58. Pace A, Savarese A, Picardo M, Maresca V, Pacetti U, Del Monte $G$, et al. Neuroprotective effect of vitamin E supplementation in patients treated with cisplatin chemotherapy. J Clin Oncol 2003;21:927-31.

59. Jonas RC, Puckett AB, Jones DP, Griffith DP, Szeszycki EE, Bergman GF, et al. Plasma antioxidant status after high-dose chemotherapy: a randomized trial of parenteral nutrition in bone marrow transplantation patients. Am J Clin Nutr 2000;72:181-9.

60. High KP, Legault C, Sinclair JA, Cruz J, Hill K, Hurd DD. Low plasma concentrations of retinol and alpha-tocopherol in hematopoietic stem cell transplant recipients: the effect of mucositis and the risk of infection. Am J Clin Nutr 2002;76:1358-66.

61. McMillan DC, Talwar D, Sattar N, Underwood M, O Reilly DS, McArdle $C$. The relationship between reduced vitamin antioxidant concentrations and the systemic inflammatory response in patients with common solid tumours. Clin Nutr 2002;21:161-4.

62. Mayland C, Allen KR, Degg TJ, Bennet M. Micronutrient concentrations in patients with malignant disease: effect of the inflammatory response. Ann Clin Biochem 2004;41:138-41.

63. Holeckova P, Krcmova L, Kalabova H, Kasparova M, Plisek J, Pala $M$, et al. Prognostic significance of serum retinol, serum alphatocopherol, and urinary neopterin in patients with head and neck carcinoma treated with external beam radiation. Int J Vitam Nutr Res 2012;82:77-84. 